\title{
Dynamo generated toroidal magnetic fields in rapidly rotating stars
}

\author{
D. Moss ${ }^{1}$ and D. Sokoloff ${ }^{2}$
}

\author{
1 School of Mathematics, University of Manchester, Oxford Road, Manchester, M13 9PL, UK \\ e-mail: moss@ma.man.ac.uk \\ 2 Department of Physics, Moscow University, 119992 Moscow, Russia \\ e-mail: sokoloff@dds.srcc.msu.su
}

Received 26 November 2008 / Accepted 16 February 2009

\begin{abstract}
Aims. Results presented in the recent observational paper by Petit et al. (2008, MNRAS, 388, 80) suggest that, for solar-like stars, the large-scale surface toroidal fields become strong for rotational periods less than about $12 \mathrm{~d}$. We discuss this observation in the context of stellar dynamo theory, as a manifestation of a bifurcation in dynamo regimes occurring around this rotation period.

Methods. Working in the context of mean field theory, we first consider two options for such a bifurcation for a given stellar rotation law: (a) a bifurcation resulting in a sudden increase of the near-surface toroidal field, with all other details of the model being kept unaltered; (b) a new type of surface boundary condition at the stellar surface, forced by internal reorganization of magnetic field with increasing rotation rate, which causes a more-or-less abrupt increase in large-scale surface toroidal field.

Results. Neither of these options seem to provide anything like the reported behaviour of surface toroidal field for plausible choices of parameters, although we cannot conclusively eliminate a possible role for the latter mechanism. We conclude that any such bifurcation most plausibly is associated with some reorganization in the stellar hydrodynamics as the stellar rotation rate increases. The simplest suggestion, i.e. a transition from a solar-like rotation law (with spoke-like angular velocity contours through the bulk of the convection zone) to a law with quasi-cylindrical isorotation contours as thought to be appropriate to rapidly rotating stars, seems to reproduce the observed phenomenology reasonably well.

Conclusions. While we appreciate the manifold uncertainties in the available theory and observations, we thus suggest that the bifurcation deduced by Petit et al. (2008) is an observational manifestation of the transition between solar-like and quasi-cylindrical rotation laws, occuring near a rotational period of $12 \mathrm{~d}$.
\end{abstract}

Key words. Sun: activity - Sun: magnetic fields - stars: magnetic fields - magnetic fields

\section{Introduction}

Historically the solar activity cycle was identified from sunspot data. Contemporary interpretation of the cycle identifies it as a magnetic phenomenon which involves toroidal as well as poloidal magnetic field components, where sunspots are considered as tracers for the toroidal magnetic field. Correspondingly, confrontation between theoretical findings and solar phenomenology based on sunspot data can only illuminate the situation from a very limited viewpoint. Specific dynamo models for the solar cycle suggest various relations between toroidal and poloidal dynamo generated solar magnetic fields (for example, phase shift between the components) which seem on first sight to be very attractive for confrontation between theory and observations. In practice however the available tracers for toroidal and poloidal solar magnetic fields are so physically different that their confrontation is quite nontrivial, and comparison between theory and observations is usually based primarily on the sunspot data. As far as it is known, the description of the solar cycle determined from data concerning tracers of poloidal magnetic field appears to be substantially different than the conventional description based on sunspot data (e.g. Obridko et al. 2006): specifically, the activity wave identified by the surface mean magnetic field appears as a system of standing waves rather than the traveling wave identified from the sunspot data.

Because the physical nature of stellar activity cycles appears predominantly similar to that of the solar cycle, it is natural to consider the relation between toroidal and poloidal magnetic fields in a wider stellar perspective. The observational identifications of toroidal and poloidal stellar magnetic components are then, however, even more indirect than those for the Sun. Suitable observational results concerning the relation between toroidal and poloidal magnetic components are obviously desirable to clarify the general situation; the recent paper by Petit et al. (2008) seems to provide some such information. In a study of solar-like dwarfs, Petit et al. (2008) estimate that large-scale surface toroidal fields become strong for rotational periods less than about $P_{\text {rot }}=12$ d, i.e. for $\Omega_{\mathrm{eq}} \gtrsim(2-2.5) \Omega_{\odot}$. They consider the phenomenon as a bifurcation between regimes for slowly rotating stars, where the surface magnetic field is presumably predominantly poloidal, and rapidly rotating stars where the surface toroidal component becomes substantial.

Our aim here is to interpret this observational finding in the context of stellar dynamo theory. Such an interpretation is far from straightforward.

We can envisage, and discuss below, three broad possibilities; that the conventional surface boundary condition of the magnetic field needs modification (see, e.g., Moss 1977, in a rather different context), that there is a bifurcation in dynamo properties as the rotation rate (and thus dynamo number) increases, due solely to a reorganization of the dynamo (e.g. Brandenburg et al. 1989; Jennings \& Weiss 1991), or that the increase in surface toroidal field is a consequence of a change of the underlying internal hydrodynamics. These possibilities are discussed in more detail in Sects. 4.1-4.3 respectively. There 
is also the possibility that the observed surface toroidal fields might be related to coronal fields with a systematic orientation, but even so their appearance at periods of about $12 \mathrm{~d}$ still needs explanation, and plausibly could still be associated with stronger interior toroidal fields, as discussed here. Our general conclusion, perhaps more important than the discussion of any particular dynamo model, is that the bifurcation suggested by Petit et al. has the potential to be very informative when confronting stellar dynamo theories with stellar activity observations.

\section{Background}

Observations of solar-type stars suggest that

$\Delta P_{\text {rot }} \propto P_{\text {rot }}^{m}$

where $\Delta P_{\text {rot }}$ is the observed variation of rotation rate over the surface (not extending above mid-latitudes) and $m \sim 1-1.4$. Thus

$\frac{\Delta \Omega}{\Omega_{\mathrm{eq}}} \propto \Omega^{n}$

where $n \sim(-0.4) \ldots 0$, and $\Omega_{\mathrm{eq}}$ is the equatorial rotation rate.

At the same time a number of observational studies are indicating the presence of large-scale toroidal fields at the surfaces of late-type stars, and Petit et al. (2008) in a study of solar-like stars suggest the existence of a "threshold" for their appearance around $P_{\text {rot }}=12 \mathrm{~d}$. Conventional dynamo models usually take as a boundary condition $\boldsymbol{B}_{T}=0$ at the stellar surface, matching the interior field to a vacuum external field (but see Covas et al. 2005). There appear to be three immediate possible theoretical explanations of the appearance of strong surface toroidal field. It is possible that the zero boundary condition on the surface toroidal field is inappropriate, and the interior field should be fitted to a more general external force-free field - this possibility attracted attention in the $1970 \mathrm{~s}$ in the context of CP star modelling (e.g. Milsom \& Wright 1976; Moss 1977). An alternative is that there is a bifurcation in the field structure as the rotation period decreases, leading to a stronger sub-surface toroidal field which becomes unstable and erupts through the surface. A third possibility is that the underlying stellar rotation law changes from a quasi-solar form, appropriate to slower rotators, to a quasicylindrical form at higher rotation rates, also leading to a sudden increase in sub-surface toroidal field strength. We study all the above possibilities in the framework of a conventional meanfield dynamo model in a stellar convective shell. Note that we thus only discuss the large-scale magnetic field.

\section{Rotation laws}

If we take standard $\alpha \Omega$ dynamo theory, then $R_{\omega} \propto \Delta \Omega \propto \Omega^{n+1}$ and $R_{\alpha} \propto \Omega$. We can construct a series of "solar type" dynamo models, with the convection zone occupying approximately the outer $30 \%$ by radius of the star, and $\Omega$ increasing from $\Omega_{\odot}$. Taking a "solar-like" surface rotation law of the form

$\Omega_{\mathrm{S}}=\Omega_{\mathrm{eq}}\left(1-a \cos ^{2} \theta-b \cos ^{4} \theta\right)$,

where $\theta$ is polar angle and "solar" values are $a=0.126, b=$ 0.159 , (cf. Tassoul 1978) we construct a plausible extrapolation between the surface $r=R$ and the base of the overshoot region at $r=r_{0} R$ of the form

$\Omega(r, \theta)=(1-f(r)) \Omega_{\mathrm{S}}\left(\theta_{\Omega}\right)+f(r) \Omega_{\mathrm{S}}(\theta)$.
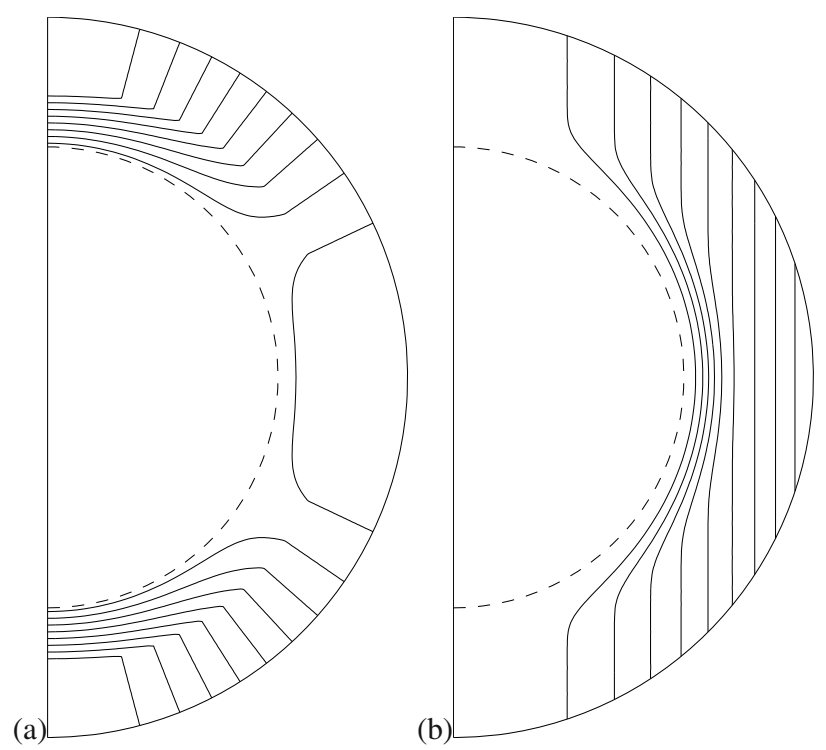

Fig. 1. Equally spaced isorotation contours for a) the solar-like rotation law; b) the quasi-cylindrical rotation law. See text for details.

Here $\theta_{\Omega}$ is taken as $\pi / 3$, and corresponds to the polar angle at which the surface angular velocity is that of the base of the tachocline, and $f(r)$ is a function that goes smoothly from unity at $r=r_{0} R$ to zero at $r=r_{\Omega} R$. We take $r_{0}=0.64, r_{\Omega}=0.80$ and $\theta_{\Omega}=\pi / 3$. Isorotation contours are shown in Fig. 1a. Thus $\Delta \Omega / \Omega_{\mathrm{eq}}=a+b=0.285$.

This value of $\Delta \Omega / \Omega_{\mathrm{eq}}$ at $\Omega=\Omega_{\odot}$ is used to determine $\Delta \Omega / \Omega_{\odot}$ for all $\Omega_{\mathrm{eq}}$ with both $n=-0.4$ and $n=0$, using (2). Although this apparently gives values that are too large compared with some observations we note (i) the Sun is the only really well-determined case; (ii) non-solar $\Delta P_{\text {rot }}$ values are estimated from a limited range of latitudes, not extending close to the poles, and so probably underestimate $\Delta \Omega / \Omega_{\mathrm{eq}}$. The stars which really seem to have very small $\Delta \Omega$ values are very rapid rotators, and so outside of the range of periods of immediate interest. In any case, if we have over-estimated $\Delta \Omega / \Omega_{\mathrm{eq}}$, then we have done so uniformly across the range of rotation periods, and any differential effects we observe should be valid.

For more rapid rotation we can expect the rotation law to be quasi-cylindrical, and so adopt as an empirical law (cf. Covas et al. 2005)

$\Omega=\frac{\left(\frac{1}{2}\left(1+\operatorname{erf}\left(\frac{r-r_{\mathrm{c}}}{d}\right)\right) f r^{2} \sin ^{2} \theta+1\right) \Omega_{\mathrm{eq}}}{1+f}$,

where $\Omega_{\mathrm{eq}}$ is the equatorial angular velocity and, rather arbitrarily, $d=0.05, r_{\mathrm{c}}=0.7 R_{*}$. Then $\Delta \Omega / \Omega_{\mathrm{eq}}=f /(1+f)$. Isorotation contours are shown in Fig. $1 \mathrm{~b}$.

\section{Results}

\subsection{Modification of surface boundary condition}

We first summarize the general theory of toroidal fields in very low density domains in the context of large-scale stellar magnetic fields. This follows quite closely the discussion in Raadu (1971), Milsom \& Wright (1976), Moss (1977). When modelling stellar magnetic fields it is necessary to ensure that the field in the interior joins smoothly onto a force-free field in the external, very low density, region. Splitting the magnetic field 


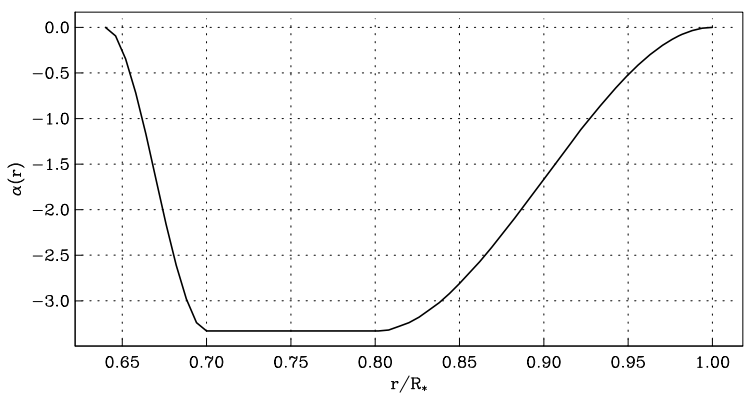

Fig. 2. The dependence of $\alpha$ on fractional radius $r / R_{*}$, at an arbitrary latitude.

into poloidal and toroidal parts, $\boldsymbol{B}=\boldsymbol{B}_{P}+\boldsymbol{B}_{T}$, the Lorentz force can be written as

$$
\begin{aligned}
\boldsymbol{L}= & \left(\nabla \times \boldsymbol{B}_{P}\right) \times \boldsymbol{B}_{P}+\left(\nabla \times \boldsymbol{B}_{T}\right) \times \boldsymbol{B}_{T} \\
& +\left(\nabla \times \boldsymbol{B}_{T}\right) \times \boldsymbol{B}_{P}+\left(\nabla \times \boldsymbol{B}_{P}\right) \times \boldsymbol{B}_{T} .
\end{aligned}
$$

Restricting consideration to axisymmetric fields, the last term is identically zero, the first two are poloidal vectors and the third is toroidal. In this case $\boldsymbol{B}_{T}=B_{\phi}$.

The condition $\boldsymbol{L}=0$ can be satisfied by setting $\boldsymbol{B}_{T}=0$ and $\nabla \times \boldsymbol{B}_{P}=0$; this provides the usual boundary condition on the interior field at $r=R$. Configurations with $\boldsymbol{L}=0$ and $\boldsymbol{B}_{T} \neq 0$ are also possible. Then a necessary condition is that $\boldsymbol{L}_{T}=\left(\nabla \times \boldsymbol{B}_{T}\right) \times \boldsymbol{B}_{T}=0$, which is the torque-free condition. If $\psi$ is the "streamfunction" for the poloidal field (so $\psi=r \sin \theta A_{\phi}$, where $A_{\phi}$ is the toroidal component of the vector potential, $\boldsymbol{B}_{P}=$ $\nabla \times\left(A_{\phi} \hat{\phi}\right)$, so that $\psi=$ constant on fieldlines of poloidal field), then $\boldsymbol{L}_{T}=0$ implies

$B_{T}=\frac{F(\psi)}{r \sin \theta}$,

where $F$ is an arbitrary function (e.g. Lüst \& Schlüter 1954). For example, Raadu (1971) showed that if $F(\psi)=K \psi^{k}$, then $k>2$ for satisfactory behaviour at large distances. Thus the boundary condition $\boldsymbol{B}_{T}=0$ at $r=R$ could be replaced by (7) - of course the vacuum condition on the poloidal field would also need amendment to give $\boldsymbol{L}_{P}=0$ with $\boldsymbol{B}_{T} \neq 0$. Moss (1977) constructed models with steady fields relevant to magnetic CP stars with such boundary conditions, by arbitrarily specifying $F(\psi)$ and solving the equation $\boldsymbol{L}_{P}=0$ explicitly in $r>R$.

This sort of formalism appears to work reasonably well when investigating the time independent field structures of the $\mathrm{CP}$ stars, but encounters difficulties when considering oscillatory dynamo fields - there are difficult physical issues connected with the properties of a time-varying external toroidal field extending to large distances. Moreover, in the case being discussed, there is a direct link between toroidal and poloidal field components via the alpha term in the dynamo equation. For example, a dipole-like dynamo field (odd parity) has $\psi$ an even function with respect to the equator, whereas $B_{\phi}$ is odd (given that $\alpha$ is antisymmetric). But then any plausible $F(\psi)$ in (7) insists that $B_{\phi}$ be an even function, in contradiction to the assumption of an odd parity field structure. It is, of course, quite implausible that the very low density environs of a late-type star can alter significantly the gross structure of the internal magnetic field ("the tail does not wag the dog"). We conclude that the analysis is incomplete.

Putting all this on one side, the pragmatic question here is whether use of boundary condition such as (7) with the corresponding amendment to the poloidal field condition would
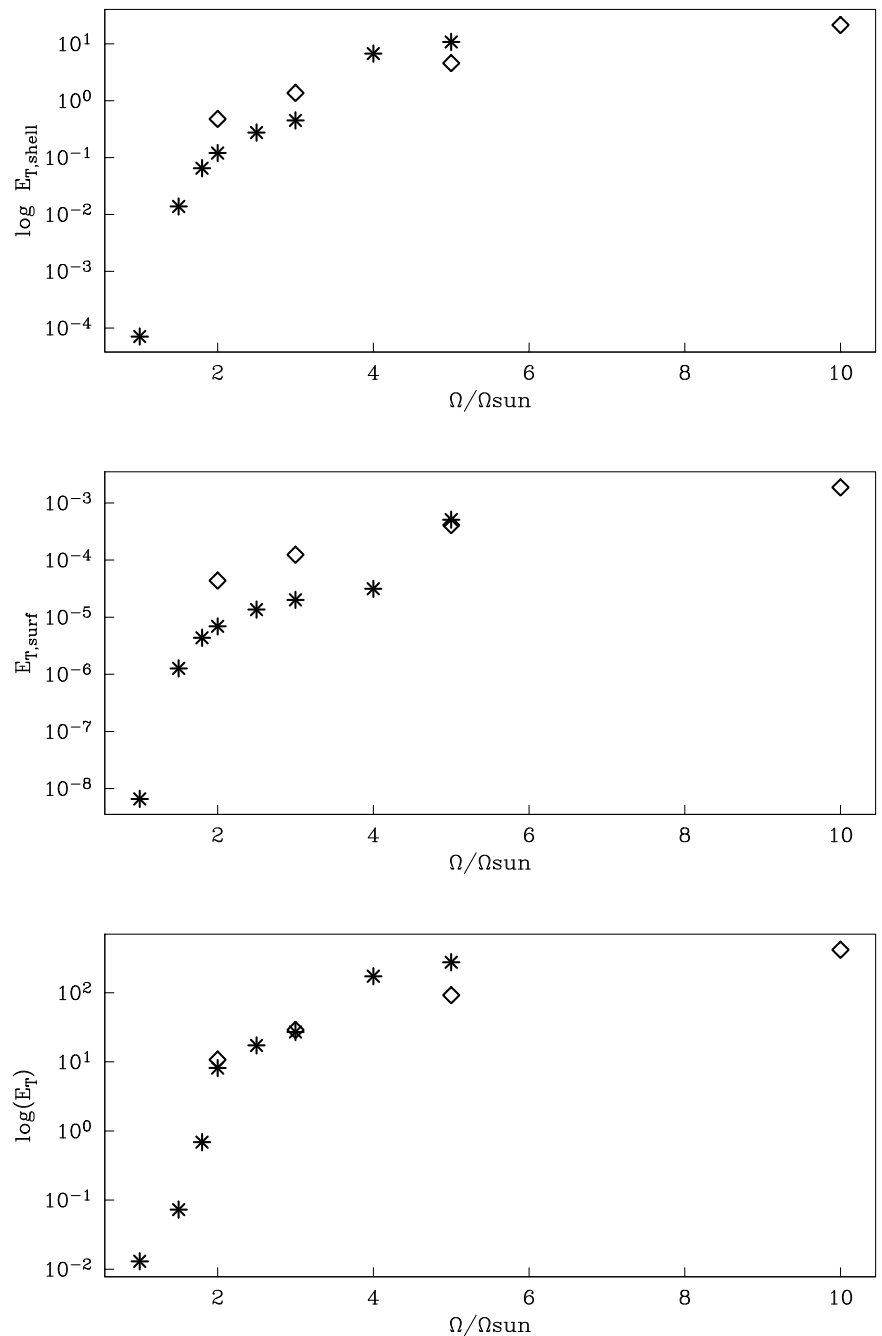

Fig. 3. Dependence of measures of toroidal energy on rotation rate with fixed parity $P=-1$ for $n=0$. Asterisks denote results with the solarlike rotation law (3), diamonds those with the quasi-cylindrical law (4).

change significantly the dependence of surface toroidal field on rotation rate. Very limited and approximate numerical experimentation (ignoring the modification to the poloidal boundary condition) did not support any strong effect, and a priori it is difficult to think of a strong physical argument that this would happen. Thus we did not pursue the matter further.

In summary, while it is clear that the surface boundary condition $B_{\phi}=0$ may be inappropriate, rigorous implementation of a more general boundary condition in the context of an oscillatory dynamo generated field requires resolution of difficult issues, which we have not pursued. Our intuition is that these issues may not be directly relevant to the problem addressed in this paper, but of course we cannot rule out such a possibility.

\subsection{Bifurcation due to more intensive dynamo action}

We use the mean field dynamo model of Covas et al. (2005), implementing a naive alpha-quenching nonlinearity rather than the feedback of the Lorentz force on the differential rotation as used there. We write $\alpha(r, \theta)=\alpha_{0}(r) \sin ^{2} \theta \cos \theta$, where $\alpha_{0}(r)$ is given in Fig. 2. The turbulent diffusivity $\eta(r)$ is taken as a constant, $\eta_{0}$, except in fractional radii $r_{0} \leq r / R_{*}<0.70$, where it changes smoothly so that $\eta\left(r_{0}\right)=0.5 \eta_{0}$. We start with a "solar" model that is about $20 \%$ supercritical. Taking $\eta_{0} \sim 3 \times 10^{11} \mathrm{~cm}^{2} \mathrm{~s}^{-1}$ 

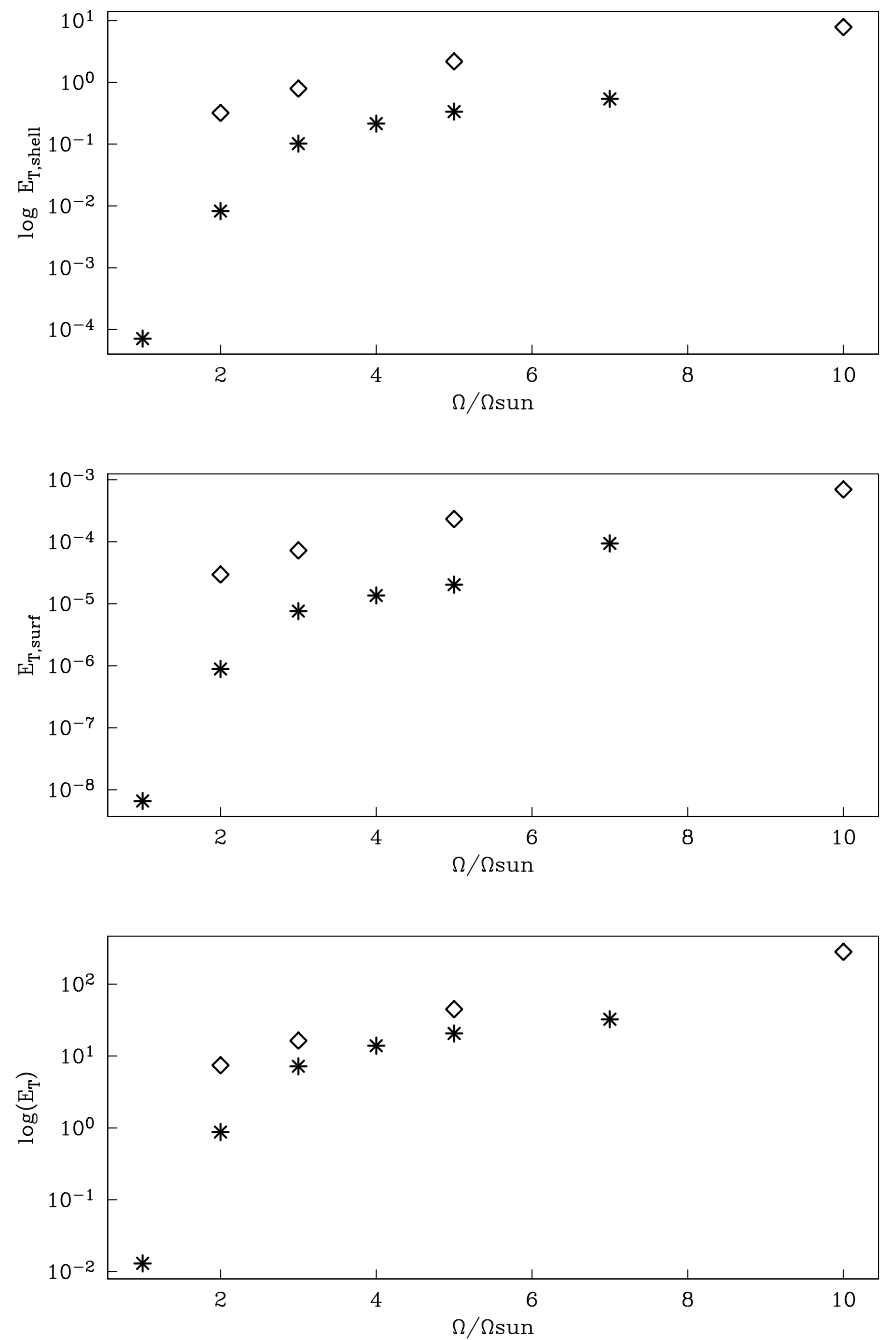

Fig. 4. Dependence of measures of toroidal energy on rotation rate with fixed parity $P=-1$ for $n=-0.4$. Notation is as in Fig. 3 .

gives the dynamo number $R_{\omega}=R^{2} \Omega_{\mathrm{eq}} / \eta_{0}=4.4 \times 10^{4}$. The marginal value of $R_{\alpha}=\alpha_{0} R / \eta_{0}$ is then about -3.2 , and so we take $R_{\alpha}=-3.9$ for this baseline model. Values of $R_{\alpha}, R_{\omega}$ for larger values of $\Omega_{\mathrm{eq}}$ are determined by scaling from these values. For each model we calculate the energies in the poloidal and toroidal components of the (axisymmetric) magnetic field in the entire dynamo-active region, the energies in the shell $0.90 \leq r / R_{*} \leq 1$, and the energies in the immediately subsurface region, $0.994 \leq r / R_{*} \leq 1.0$, respectively $E_{P}, E_{T}, E_{P \text {, shell }}$, $E_{T \text {,shell }}, E_{P \text {,surf }}, E_{T \text {,surf }}$. Specifically calculations were performed for the solar-like rotation law for $1 \leq \Omega_{\mathrm{eq}} / \Omega_{\odot} \leq 7$, and for the quasi-cylindrical rotation law with $1 \leq \Omega_{\mathrm{eq}} / \Omega_{\odot} \leq 20$, for both $n=-0.4$ and $n=0$.

These computations were all performed with fixed (dipolelike) parity of the magnetic fields, i.e. $P=-1$ (where $P=+1$ indicates the opposing, quadrupole-like, configuration, see e.g. Brandenburg et al. 1989). See Figs. 3 and 4. We then repeated the computations for the case $n=-0.4$, allowing full freedom for bifurcations in field structure to appear. We started the computations from initial fields with intermediate parity $P=0$, and evolved the field until a stable configuration had been attained. As expected, in some cases the eventual state did not have $P=-1,-$ see Table 1 for the rotation law (3). Models with the rotation law (4) all had a stable state with $P=+1$. However the mean field energies in the final state did not differ radically from
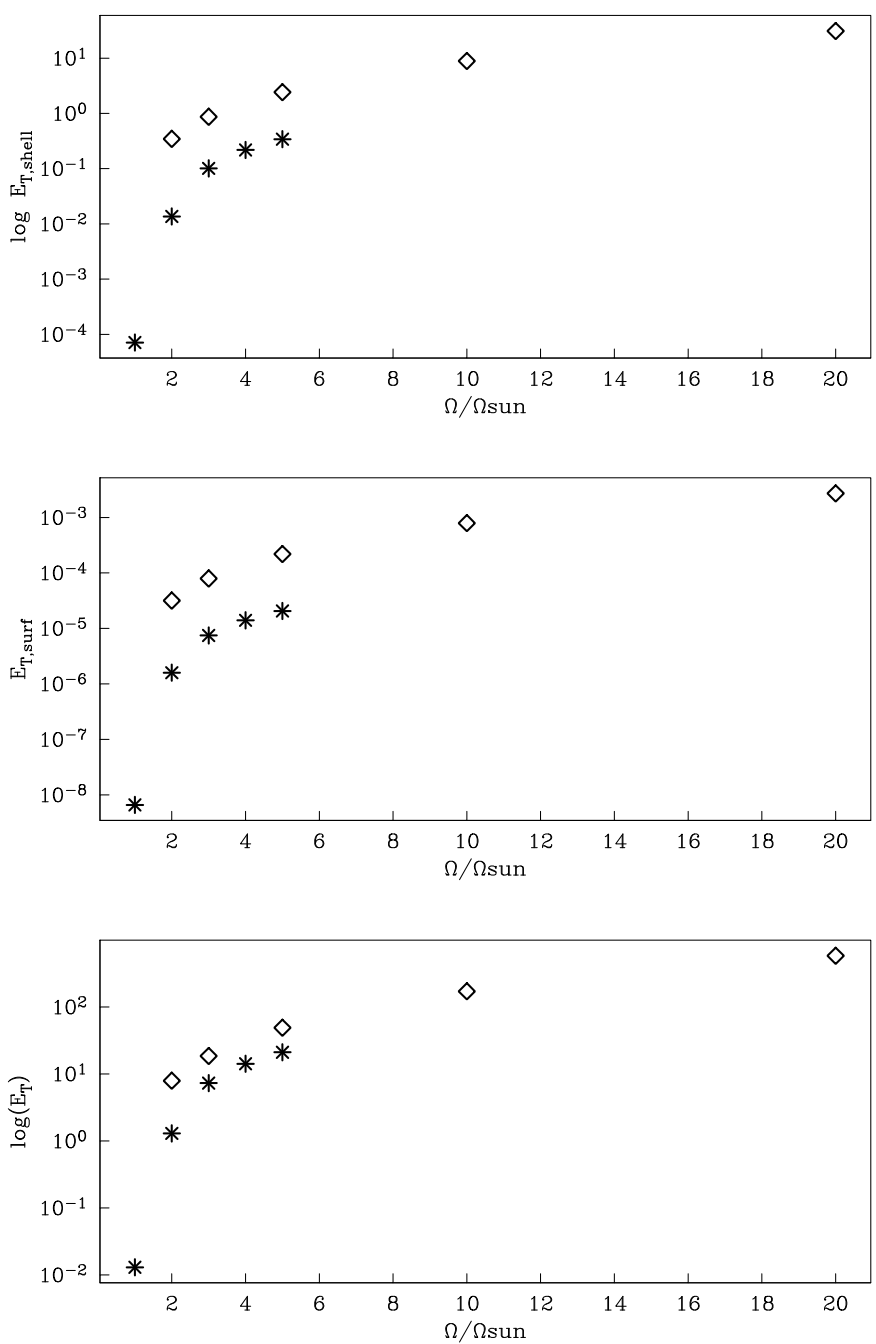

Fig. 5. Dependence of measures of toroidal energy on rotation rate with free parity for $n=-0.4$. Notation is as in Fig. 3 .

Table 1. Mean parity versus rotation rate for models with solar-like rotation-law and $\Delta \Omega \propto \Omega^{-0.4}$. All models are singly periodic.

\begin{tabular}{cccc}
\hline \hline$\Omega / \Omega_{\odot}$ & $\langle P\rangle$ & $\Omega / \Omega_{\odot}$ & $\langle P\rangle$ \\
\hline 1 & -1.0 & 4 & -0.86 \\
2 & -1.0 & 5 & -0.88 \\
3 & -0.80 & 7 & -1.0 \\
\hline
\end{tabular}

those determined previously when $P=-1$ was imposed, and the plots of field energies against equatorial angular velocity, shown in Fig. 5, do not look significantly different to those in Figs. 3 and 4.

When we examine results for a fixed rotation law (i.e. only diamonds or only asterisks in the figures), we see no evidence for a sudden bifurcation, e.g. to a branch with markedly higher energies, as the rotation speed increases. The only exception is in Fig. 3 (rotation law (3)) at $\Omega_{\mathrm{eq}} / \Omega_{\odot} \gtrsim 4$. However the magnetic fields of the models with $\Omega_{\mathrm{eq}} / \Omega_{\odot}=4,5$ are steady, and so irrelevant to our investigation.

\subsection{Transition from one rotation law to the other}

If we now consider the results for the two rotation laws together, the graphs of $E_{T \text {,surf }}$ for both $n=0$ and $n=-0.4$ show a 
displacement by a factor of about 4 at $\Omega_{\mathrm{eq}}=(2-3) \Omega_{\odot}$, and there is a similar, smaller, discontinuity in the graphs for $E_{T \text {,shell, }}$ see Figs. 3 and 4. The other measures of magnetic energy are almost continuous when switching from one rotation law to the other.

Thus Figs. 3-5 give a hint that the bifurcation under discussion can be associated with a transition from a solar-like rotation law with approximately radial contours through most of the convection zone to a quasi-cylindrical law. Of course in "reality" a gradual transition from a rotation law appropriate to slow rotators to a rapid-rotator law could be expected. However, unless the transition is over a wide range of rotation speeds, the above treatment should be adequate to illustrate the behaviour of field energies as $\Omega_{\mathrm{eq}}$ increases. In principle, it would be trivial to compose a synthetic rotation law that was solar-like for say $\Omega<0.9 \Omega_{\text {eqc }}$ and quasi-cylindrical for $\Omega>1.1 \Omega_{\text {eqc }}$, where $\Omega_{\text {eqc }} \sim 12 \mathrm{~d}$, with a smooth transition between. Then our results presented in Sect. 4.2 would be valid outside of this transition range, and would suggest a significant jump in toroidal field, especially in the measures $E_{T \text {,surf }}$ and $E_{T \text {,shell }}$, as the rotation law changes.

\section{Discussion}

Our results presented in Figs. 3-5 show that there is no evidence for marked bifurcations in magnetic energy for given hydrodynamic flows that do not change with rotation rate - i.e. the relations defined by the asterisks and diamonds separately do not show any sudden changes of slope (except for the two most rapidly rotating models of Fig. 3 with the solar-like rotation law, where there has been a bifurcation from oscillatory to (physically irrelevant) steady solutions).
On the other hand there is a distinct jump in the large-scale toroidal energy between the relations for solar-like and quasicylindrical rotational laws. If we believe that these rotation laws are more-or-less appropriate physically, then we have a possibility of identifying the origin of the observed appearance of largescale surface toroidal fields around $P_{\text {rot }}=12 \mathrm{~d}$, namely that it is a result of a change in the internal azimuthal flows, as the TaylorProudman constraint becomes important at shorter rotational periods. We realise that our conclusions may be rather model dependent, but would stress in conclusion our belief that observations such as those of Petit et al. (2008) have considerable potential for illuminating and discriminating between various mechanisms for magnetic field generation.

Acknowledgements. D.S. is grateful to financial support from RFBR under grant 07-02-00127a. We thank the referee, A. Brandenburg, for his constructive comments.

\section{References}

Brandenburg, A., Krause, F., Meinel, R., Moss, D., \& Tuominen, I. 1989, A\&A, 213,411

Covas, E., Moss, D., \& Tavakol, R. 2005, A\&A, 429, 657

Jennings, R. L., \& Weiss, N. O. 1991, MNRAS, 252, 249

Lüst, R., \& Schlüter, A. 1954, Z. Astrophys., 34, 263

Milsom, F., \& Wright, G. A. 1976, MNRAS, 174, 307

Moss, D. 1977, MNRAS, 178, 51

Obridko, V. N., Sokoloff, D. D., Kuzanyan, K. M., Shelting, B. D., \& Zakharov, V. G. 2006, MNRAS, 365, 827

Petit, P., Dintrans, B., Solanki, S. K., et al. 2008, MNRAS, 388, 80

Tassoul, J.-L. 1978, Theory of Rotating Stars (Princeton, N.J.: Princeton Univ. Press)

Raadu, M. 1971, Ap\&SS, 14, 464 